\title{
Reassessing the HPI: The Chronology of Present Illness (CPI)
}

\author{
Kelley M. Skeff, MD, PhD \\ Stanford University Medical School, Stanford, CA, USA. \\ $\mathrm{J}$ Gen Intern Med 29(1):13-5 \\ DOI: $10.1007 / \mathrm{s} 11606-013-2573-3$ \\ (c) Society of General Internal Medicine 2013
}

\section{BACKGROUND}

The history of present illness provides the initial data to generate the differential diagnoses, guide medical decisionmaking, investigate the patient's problem, and ultimately analyze the patient's illness. Yet, physician-teachers often hear or read a patient's history that is not clear. Why are histories difficult to obtain, present, and analyze? Admittedly, it can be the complexity of the case or the challenges in acquiring and relating the patient's story. However, an unclear history frequently exists because of the lack of an overt chronological structure used to organize the patient's story.

In this paper, I present a strategy - the Chronology of Present Illness (CPI) - that emphasizes time as the core structural element. It is simple, clarifying, and often enables the trainee or fully trained physician to not only effectively take and report a comprehensive and relevant history, but also be a more effective diagnostician. Although sometimes used in complex clinical areas, such as oncology and intensive care units, it is seldom taught as a preferred approach for taking and communicating the patient's history. In over three decades of my teaching this method, it has consistently appeared "new" to students and houseofficers. Therefore, I have chosen to publish it for consideration by teachers and trainees as a model for patient histories deserving further study.

\section{PROPOSED SOLUTION}

\section{The CPI-Chronology of Present Illness: Emphasizing Time}

Currently, most students and housestaff are taught to write or present the History of Present Illness (HPI) in prose, telling the patient's history as a story. This narrative style may be understandably chosen to demonstrate respect for the patient's story while increasing physician flexibility in documentation. However, these benefits may be

Published online August 16, 2013 overshadowed by variability in the gathering, presentation, and analysis of the crucial factual data related to the patient's medical history.

Whereas histories in prose have references to time imbedded within sentences, the CPI explicitly uses time as the main organizational variable for documenting the evolution of the patient's illness, overtly identifying times when symptoms appeared or changed. In CPI notes, the dates or times are set out in the left-hand side of the page, with description of the symptoms, concurrent patient activities, treatments, responses, etc. on the corresponding right-hand side. In my opinion, for each case, the times can be either actual (January, 2011) or relative to the patient's admission ( 3 days prior to admission). Symptom description can be in paragraphs, sentences, or, if adequate to communicate the symptoms, brief bullet points. This written structure enables the writer and readers to use visual and verbal abilities to comprehend the time course of the disease. Oral presentations can also highlight time to underscore disease evolution. Table 1 illustrates the contrast between a time-oriented CPI and an actual prose HPI, demonstrating the resultant brevity and clarity that can be gained.

\section{DISCUSSION}

Emphasizing chronology in understanding the patient's illness is not new. However, we have inadequately emphasized the utility of a discernible timeline that provides a cognitive structure that facilitates clinicians' abilities to collect, document, analyze, and report the nuances of disease evolution. Diligently documenting the historical timeline can effectively reveal clues to the pathophysiological process and diminish the likelihood of either ignoring or overemphasizing patient's symptoms. Deserving of formal study, this structure may enhance the completeness, consistency, fidelity, clarity, accuracy, and brevity of the historical data. Of note, it should neither diminish the desired patient-centered openness and sensitivity in listening to and documenting the patient's story, nor de-emphasize the patient's interpretation or emotional response to illness. 
Table 1. HPI/CPI Comparison

\begin{tabular}{lc}
\hline HPI Example CPI Example & CPam
\end{tabular}

Patient is a 32yo M with PMH significant for anal fissures $\mathrm{s} / \mathrm{p}$ fissurectomy on $7 / 26 / 10$, now presenting with continued diarrhea with anal pain, new onset upper abdominal pain with nausea, and subjective fevers. Patient was in his usual state of health until patient travelled abroad in May 2010. He notes a change in diet, most significant for high-meat and high-EtOH, and returned with 1.5 weeks of diarrhea with minimal blood on toilet paper that self-resolved on initiation of his usual diet. He again returned overseas in June 2010 with similar dietary indiscretions, and had again the onset of diarrhea that did not resolve on his return. Two weeks later, the patient also noted significant rectal pain with defecation described as "sore and tender,"

He went to see his PCP on 7/13/10 after finding more blood on the toilet paper after wiping, who prescribed vicodin and Metamucil. He then saw Dr. X (GI) on $7 / 20 / 10$, who started him on a 10 -day course of hydrocortisone suppositories with minimal relief. He was referred to Dr. Y (colorectal surgery) on $7 / 23 / 10$ who recommended use of nitroglycerin ointment in the rectum. Dr. Y then performed an exam under anesthesia on $7 / 26 / 10$, finding anterior and posterior ulcerations, with biopsy finding granulomatous tissue with granulomas and focal ulceration with lymphoid aggregates. He also performed a fissurectomy at that time. Given the patient continued to have fevers, he was started on Flagyl 250 PO BID on 8/3/10, but on noting a "rash" of his bilateral shins, called a physician and was instructed to discontinue flagyl on $8 / 15 / 10$, without interval resolution of his skin findings. Also, of note, the patient strained his back on 8/12/10 while getting out of a sitz bath, and had significant lumbar back pain and spasms, improved with valium $5 \mathrm{mg}$ PO BID PRN, now rated 3/10 with movement.

The patient now acutely presented to the ED with severe bilateral upper abdominal pain since 1900 on $8 / 18 / 10$ described as a "nausea" pain, that has a constant baseline level of pain with colicky exacerbations lasting 20 seconds. The patient is unable to identify any exacerbating or alleviating factors (including defecation), with the exception that lying supine helps relieve the pain. This is associated with fever to $100 \mathrm{~F}$, severe nausea, a burning sensation from the epigastrium up along the esophagus, and belching. in the ED. While in the ED, the patient was also given a GI cocktail and resulted in 1 episode of non-bloody emesis. Of note, patient states he ate a burrito with cheese and black beans several hours prior to the onset of the abdominal pain, and has not had these food in quite a while. He states a childhood history of lactose intolerance, and has recently been avoiding dairy as he feels this exacerbates his diarrhea.

Patient continues to have 4-5 loose watery stools daily, with significant pain rated 5-10/10 on defecation with blood on the toilet paper and surface of the stool. He also complains of a very swollen anus causing significant discomfort and a constant "pinching" sensation of his anus rated $3 / 10$. He also notes decreased appetite, fatigue, 15 pound weight loss, and weakness over the last month. Denies sick contacts.
Patient is a 32yo M with PMH significant for anal fissures and diarrhea, presenting with new onset abdominal pain, nausea, and subjective fevers.

Patient was in his usual state of health until:

May 2010:

Travelled abroad, diet change (high meat and high Etoh). On return to US, had 1.5 weeks of diarrhea with blood tinged BMs, resolved with usual diet.

\section{June 2010:}

Returned overseas with same diet, noted diarrhea that did not resolve on return. Also developed rectal pain on defecation.

July 13, 2010: July 20: Blood on toilet paper, so saw PCP. Rx: vicodin/Metamucil.

Saw GI physician, Dr. X. Rx - 10 day HC suppositories with minimal relief.

July 23:

\author{
Saw colorectal surgeon, rx nitroglycerin suppositories;
}

July 26:

Exam under anesthesia; findings included anterior and posterior ulcerations; bx showed granulomas, focal ulceration with lymphoid aggregates. Also performed fissurectomy.

August 3, 2010:

Ongoing fevers, rx flagyl $250 \mathrm{BID}$, later rash on shins, thus, DC flagyl on $8 / 15 / 10$. Rash persisted.

August 12:

Back strain on moving from sitz bath, improved with valium.

Ongoing 4-5 loose watery stools daily, with pain on defecation, blood on the toilet paper and surface of the stool. C/O swollen painful anus with "pinching" sensation. Decreased appetite, fatigue, 15 pound weight loss, and weakness over the last month. Denies sick contacts. Has avoided dairy because of lactose intolerance;

August 18: 7PM:

New onset severe, bilateral upper abdominal pain, constant baseline with colicky exacerbations lasting 20 seconds. Pain preceded by eating Mexican food (burrito/cheese/beans) several hours before. No exacerbating factors; relieved by lying supine. Pain associated with fever to $100 \mathrm{~F}$, severe nausea, burning sensation from epigastrium up along esophagus, belching.

Presented to ED. Pain relieved in ED by morphine and Zofran. GI cocktail $->$ non-bloody emesis. Admitted because of persistent symptoms and unclear diagnoses.
The CPI has potential benefits for physicians, health care systems, trainees, teachers, and patients. For all physicians, this systematically organized and time-oriented data collection and documentation may improve the quality of the data for the initial problem representation for medical decisionmaking. As noted by Gruppen and Frohna, this initial problem representation provides the foundation for the rest of the reasoning process. ${ }^{1}$ The resultant clarity can facilitate the clinical reasoning approaches for both pattern recognition and deliberate analysis. Moreover, a clear illness chronology can promote ongoing physician learning. By adhering to the symptom timeline, subtle and puzzling patient symptoms are identified and exposed for consideration and analysis, stimulating consultation or literature review that can reveal the diagnostic meaning of the unusual or unrecognized symptom evolution.
The CPI may also save time by enabling more efficient documentation, review, and analysis of patient histories, benefitting the entire health care team. This is especially important in today's world of cross-coverage, shift-work, and Relative Value Unit (RVU) reimbursement. Time-oriented notes can facilitate writing and revising sections of notes without restructuring entire paragraphs, thus diminishing the temptation to "cut-and-paste" entire notes. By facilitating all providers' focused review of chronology, the CPI may facilitate corrections, reducing the possibility of "carry forward" imbedded historical errors across admissions. Moreover, other team members, colleagues, trainees, and chart coders can glean a quicker understanding of the patient's illness when needed for consultation, cross coverage, and chart review.

Trainees may benefit from this method. Through clear timeline-oriented histories, students can be more effective 
reporters, interpreters, and even educators by presenting clearly to others, thus improving several stages in the Reporter-Interpreter-Manager-Educator scheme. ${ }^{2}$ For more advanced trainees, a CPI timeline may facilitate increased focus on nuances and variation in patient histories as evolving patient symptomatology is systematically and chronologically documented. This may lead to an earlier recognition of illness scripts and their variations.

For clinical educators, the chronological structure can facilitate the teacher's evaluation of the trainee's data collection and analysis, allowing identification of time-gaps in the patient history or erroneous interpretation of symptoms, thereby, assisting clinical teachers in the first step for efficient teaching defined by Irby and Wilkerson, identifying learner needs. ${ }^{3}$ With timeline clarity, teachers can efficiently crosscheck historical data, investigate time gaps, and focus discussion on different interpretations of symptom evolution. This stepwise analysis enables coaching as described by Kassirer, examining the thought process as the symptoms evolve. ${ }^{4}$ Through this standardized structure, the CPI may diminish the occurrence of the changing history with different historians, allowing more time to be spent on understanding the illness instead of history clarification.

Finally, patients may benefit from the CPI model. The literature emphasizes patients' difficulty in relating histories. ${ }^{5}$ The CPI can assist the patient in providing the history by using an approach familiar to patients - relating life's experiences in terms of time. This approach should neither require that the history taker interrupt or prevent the patient's ability to relate his or her story, nor disregard the personal human or emotional aspects of the patient's history. However, with chronological guidance, patients can be assisted to relate a more complete and coherent history for analysis. Requests such as "please describe your very first symptom" or "when and how did it change" can assist the patient to provide critical diagnostic information for the physician. By overtly seeking factual clarity in the patient's story, we not only can convey interest and trust, we can also enable the physician to have quality and systematic data for diagnostic analysis for the patient's benefit. The Chronology of Present Illness can advance this cause, and is therefore deserving of further consideration and study.

Acknowledgements: The author would like to thank multiple faculty for reviewing this manuscript, but most of all to thank the Stanford medical students and housestaff whose opinions regarding the utility of this method stimulated me to write this article. Special thanks go to Dr. Matthew Goldstein, who, as a Stanford medical student, suggested the term, CPI-Chronology of Present Illness, as the name for the method.

Conflict of Interest: The author declares there is no conflict of interest.

Corresponding Author: Kelley M. Skeff, $M D, P h D$; Stanford University Medical School, Medical School Office Building Room 3341265 Welch Road, Stanford, CA 94305-5475, USA (e-mail: skeff@stanford.edu).

\section{REFERENCES}

1. Gruppen LD, Frohna AZ. Clinical reasoning. In Norman GR, et al. International Handbook of Research in Medical Education, Part 1, Wolters Kluwer Academic Publishers, Norwell, MA: 2002. p 207.

2. Pangaro L. A new vocabulary and other innovations for improving descriptive in-training evaluations. Acad Med. 1999;74(11):1203-7. PMID: 10587681.

3. Irby DM, Wilkerson L. Teaching when time is limited. BMJ. 2008;336:384-7. PMID: 18276715.

4. Kassirer JP. Teaching clinical reasoning: case-based and coached. Acad Med. 2010;85:1118-24. PMID: 20603909.

5. Gillis J. The history of the patient history since 1850. Bull Hist Med. 2006;80(3):490-512. PMID: 17147133. 